\title{
FeelHippo: a low-cost autonomous underwater vehicle for subsea monitoring and inspection
}

\author{
A. Ridolfi (IEEE Member), R. Costanzi, F. Fanelli, N. Monni, B. Allotta (IEEE Member), S. Bianchi, \\ R. Conti, J. Gelli, L. Gori, L. Pugi, A. Rindi \\ Department of Industrial Engineering (DIEF) \\ University of Florence \\ Via di Santa Marta 3, 50139, Florence, Italy \\ Email: a.ridolfi@unifi.it
}

\begin{abstract}
The paper describes the development and the main characteristics of a low-cost Unmanned Underwater Vehicles (UUV) built by the Mechatronics and Dynamic Modelling Laboratory (MDM Lab) of the University of Florence. This vehicle is named FeelHippo, and it is an Autonomous Underwater Vehicle (AUV) purposely developed to participate to the 2013 edition of the Student Autonomous Underwater Vehicle Challenge-Europe (SAUC-e, http://sauc-europe.org/ ) organized by the NATO-STO Centre for Maritime Research and Experimentation (CMRE), La Spezia, Italy. SAUC-e 2013 has been a good test field for the preliminary testing of the AUV capabilities and FeelHippo ranked third in the competition. In the paper some experimental results related to the development of a low-cost vehicle localization system, suitable inside an environment a priori known, are given and discussed.
\end{abstract}

\section{INTRODUCTION}

There is a wide industrial interest for the development of off-shore power generation plants, with a particular attention to marine energy harvesting and renewable resources such as marine currents [1]. Marine power generation has several advantages, in terms of reliability and availability with respect to other renewable resources such as the solar and the Eolic ones. Additional opportunities arise from the decision of Microsoft to start the development of a new generation of sustainable underwater data centers that could provide an immediate application for the localized production of renewable marine energy. There is a wide literature concerning the usage of autonomous and remotely operated vehicles to inspect underwater plants and structures such as in the cited examples [2] [3]. This paper describes some experiences gained by the University of Florence (UNIFI) on the development of a low-cost Unmanned Underwater Vehicles (UUV). This vehicle is named FeelHippo, and it is an Autonomous Underwater Vehicle (AUV) purposely developed to participate to the 2013 edition of the Student Autonomous Underwater Vehicle Challenge-Europe (SAUC-e, http://sauc-europe.org/ ) organized by the NATO-STO Centre for Maritime Research and Experimentation (CMRE), La Spezia, Italy. FeelHippo can be used both as an AUV and as a Remotely Operated Vehicle (ROV), depending on the task at hand. It is a low-cost mobile robot (its basic cost is around $13000 €$, less than a small commercial ROV). Thanks to its small size and its high maneuverability, FeelHippo AUV is able to perform monitoring and patrolling tasks and may result useful in various scientific applications, e.g. the inspection of off-shore power generation plants. In particular, its reduced encumbrances and its high maneuverability, make FeelHippo ideal for the inspection of small plants, even in presence of not too high currents. So it should considered an ideal solution for the inspection of small sized plant such as the ones that can be used to fed small installations such as submerged server and data centers (e.g. the one visible in Figure 1).

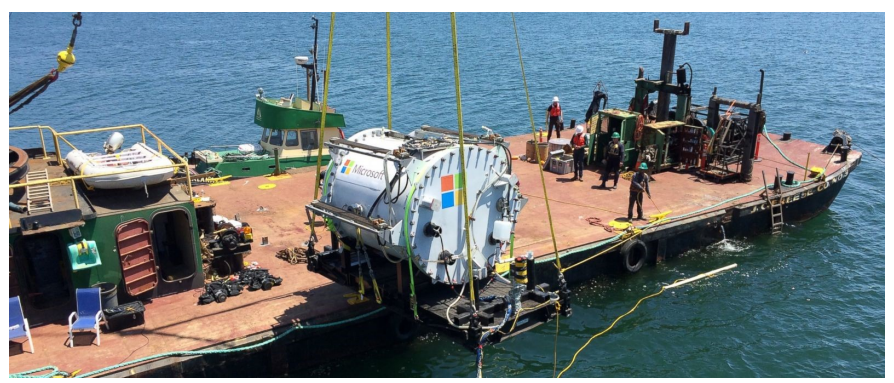

Fig. 1. Microsoft Submerged Data Center

It has several navigation sensors allowing to perform quite accurate dead reckoning, an acoustic modem for underwater communication as well as sonar and video payload to acquire data from the field.

\section{FEELHIPPO AUV DESCRIPTION}

FeelHippo AUV is capable of hovering even if it is a lowcost AUV. In fact, as this work shows, some methods can be developed in order to use cheaper devices for purposes that usually require more expensive ones. The central watertight body is made of a plexiglass pipe, closed at the sides by two domes made of the same material. The whole non-waterproof hardware, except for the batteries, is contained inside this body. Some external devices, such as the motors, are connected to six aluminum bars, which are the carrying structure of the vehicle. The power supply system is made of four LiPo batteries, stored in two aluminum cylinders under the main body. Vehicle sizes are $600 \times 640 \times 450 \mathrm{~mm}$ and its mass is about $40 \mathrm{~kg}$.

FeelHippo results to be slightly positive, ensuring it to naturally emerge in case of malfunction. The main devices onboard are:

- Power source: 4ZIPPY Flightmax six cells LiPo batteries, 22.2 nominal voltage (connected in parallel), providing a total onboard energy of about $710 \mathrm{Wh}$; 
- Computer: Odroid-XU, a small and powerful low cost computer, with Exynos5 Octa Core processor and GNU/Linux Ubuntu 14.04 installed;

- Propulsion system: 4 brushless Maxon motors (100W each) with 1:3.8 gearbox enclosed in a watertight case made by the authors [4]; these motors are driven by 4ROXXY BL-Control 930-6 motor drivers;

- $\quad$ Pressure sensor: MS5541 from Measurement Specialties is a micro sensor used to estimate the vehicle depth;

- Inertial Measurement Unit: Xsens MTi, which includes three-axial gyro, magnetic compass and accelerometer;

- $\quad$ Echo sounder: Imagenex 852, a miniature mono-axial sonar used to measure the distance from objects.

Recently FeelHippo has been substantially renovated for the participation in the robotic competition euRathlon 2015, http://www.eurathlon.eu/ (Figure 2). Three main devices, the first two ones for navigation, have been added to the vehicle:

- $\quad$ Linear velocity sensor: Doppler Velocity Log (DVL) NavQuest 600 by LinkQuest able to measure the 3D speed of the vehicle;

- $\quad$ Single axis Fiber Optic Gyro (FOG): 1 axis DSP-1760 FOG by KVH for the accurate yaw measurement;

- $\quad 2 \mathrm{D}$ forward-looking sonar (payload): Teledyne BlueView M900 acoustic device for underwater acquisition.

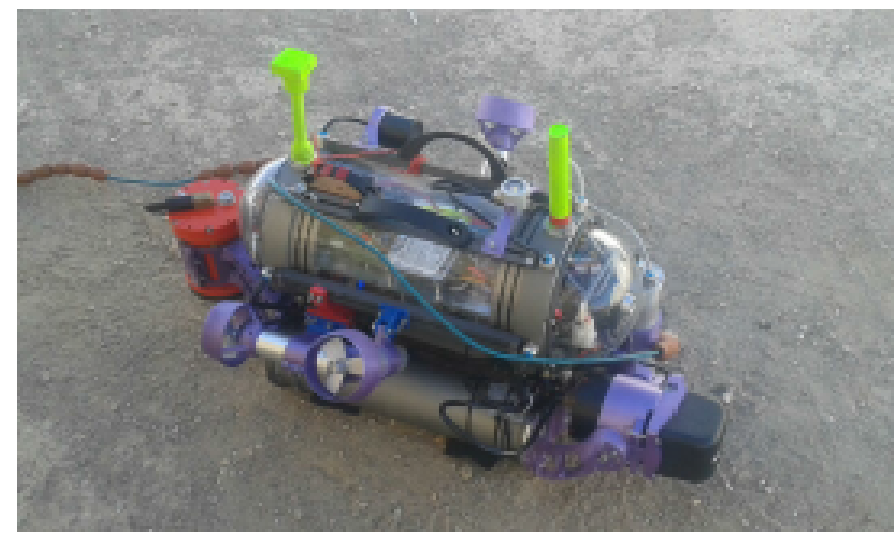

Fig. 2. FeelHippo AUV: vehicle version for the euRathlon 2015 challenge

\section{A. Propulsion system}

FeelHippo is actuated by four $100 \mathrm{~W}$ brushless motors by Maxon EC Motor, with 1:3.8 gearboxes. In order to operate underwater they have watertight aluminum cases filled with a dielectric coolant oil called Fluorinert, improving heat dissipation. The three blades propellers [5] have been built by stereolithography with a fast prototyping printer owned by MDM Lab. This propulsion system has been developed in order to control four of the six vehicle DOF, placing the four propellers as visible in Figure 2. Two of them are placed horizontally on the AUV stern and allow to control the surge and yaw motions; the other two are placed laterally in a V-shape with an angle of $\gamma$ (equal to $30^{\circ}$ ) with respect to the horizontal plane and allow to control the sway and heave motions. Roll and pitch motions are passively controlled, thanks to hydrostatic stability.

\section{B. Localization system}

FeelHippo localization system estimates the position of the vehicle on the horizontal plane, in a structured and known environment.

The Imagenex 852 Ultra Miniature Echo Sounder (Figure 3) has been mounted on top of FeelHippo bow. This kind of device can be used for different purposes in underwater robotics, e.g. [6], [7]: obstacle localization; profiling, object recognition, positioning. This work requires this device in order to estimate the 2D position of the vehicle inside a known environment, measuring the distances from the present walls.

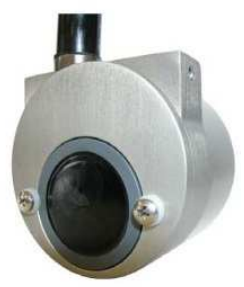

Fig. 3. Imagenex 852 Ultra Miniature Echo Sounder

As mentioned above, this kind of device is mono-axial but the vehicle needs to get measurements from every direction: a rotating support has thus been used. The support is moved by the Dynamixel AX-18A servo motor, that has a good tradeoff between dimensions and power: it allows a $300^{\circ}$ rotation angle around its axis, with a precision of $0.29^{\circ}$. It also gives position, temperature and torque feedbacks.

A CAD model of the described system is shown in Figure 4 , the echo sounder is located on top of the bow and the servo remains in the dry section of the vehicle.

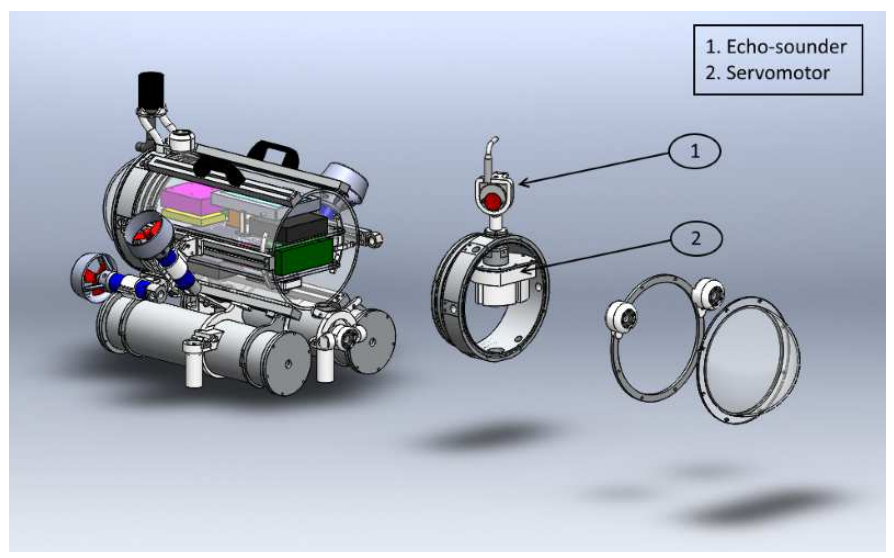

Fig. 4. Vehicle - echosounder CAD drawing

The Xsens MTi is a miniature MEMS [8] based on AHRS. An AHRS (Attitude and Heading Reference System) provides $3 \mathrm{D}$ orientation by integrating gyroscopes and fusing these data 
with accelerometer and magnetometer readings. With sensor fusion, drift from the gyroscopes integration is compensated by reference vectors, namely gravity and the Earth magnetic field. This results in a drift-free orientation, making an AHRS a more cost effective solution than conventional high-grade IMUs (Inertial Measurement Units) that only integrate gyroscopes and rely on a very high bias-stability of the gyroscopes. Thus this device produces a reliable estimation of the vehicle attitude, which can be used to drive the vehicle and also to give an Earth-fixed attitude reference to the echo sounder positioning system. In addition, the single axis FOG and a suitable algorithm can be used [9] if the vehicle has to face unkwown magnetic disturbances.

\section{Position measurement}

To locate the vehicle, with respect to an Earth-fixed reference frame, a purely geometric method is used, that requires the assumption of having knowledge of the environment where the vehicle is moving. Throughout the discussion we consider as navigation environment the pool sited in the Mechatronics and Dynamic Modelling Laboratory (MDM Lab), located in Pistoia (Italy), of the University of Florence. In this pool the proposed approach has been tested and validated.

Referring to Figure 5, a pool with the following dimensions is considered:

- lenght $L_{x}=5 \mathrm{~m}$

- $\quad$ width $L_{y}=2,5 \mathrm{~m}$

- depth $L_{z}=1 \mathrm{~m}$

with the fixed reference frame oriented as shown in the Figure. With these assumptions, the vehicle can obtain its position by means of simple geometrical relations, starting from the measurements of the distances $d_{x}$ and $d_{y}$ from two orthogonal walls.

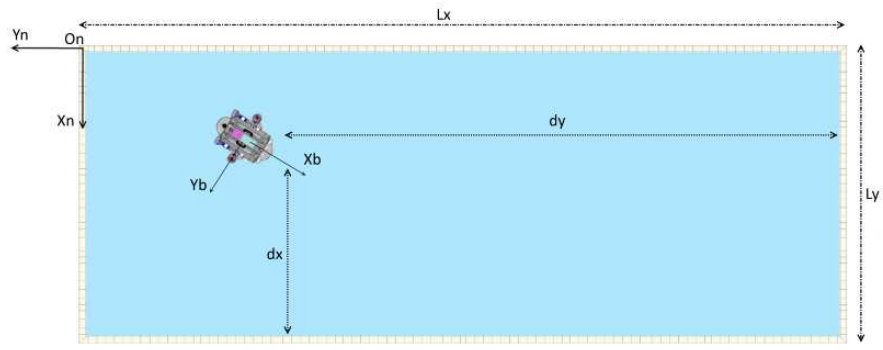

Fig. 5. MDM Lab pool used as the environment for testing and validation

The echo sounder measurement is based on the SONAR (SOund NAvigation and Ranging) technique, which uses sound propagation (usually underwater, as in submarine navigation) to navigate, communicate or detect objects on or under the surface of the water. It is necessary to define how to move the servo properly to get the measurements of the distances $d_{x}$ and $d_{y}$ from the walls. Assuming that the pool is a parallelepiped with one of the sides along the Earth frame $Z$ axis, coincident with the vehicle $Z$ axis (roll and pitch hydrostatic stability), the problem can be reduced to a $2 \mathrm{D}$ case. The idea is to define a time span $T$ while the sonar line of sight is pointing orthogonally one of the walls; when this time expires the sonar has to be pointed toward another wall orthogonal to the first one, and so on. Thanks to the IMU the attitude of the vehicle is always known, thus, by the knowledge of the environment, it is possible to write the following relation:

$\begin{cases}\psi_{s}=\psi_{p}-\psi_{b}+k \pi & \text { if } 2 n T \leq t<T(2 n+1) \\ \psi_{s}=\psi_{p}-\psi_{b}+k \pi-\frac{\pi}{2} & \text { if } T(2 n+1) \leq t<2 T(n+1)\end{cases}$

where $\psi_{s}$ is the sonar relative orientation with respect to the vehicle $x$ axis, $\psi_{p}$ is the pool orientation referred to a North East frame, $\psi_{b}$ is the vehicle yaw angle, $k$ can be 0 or $1, t$ is time and $n$ is a positive integer. There are two additional practical issues: the former is that on the upper part of the vehicle stern there is the acoustic transducer which occupies the sonar light of sight; the latter is that the servo motor range is bounded to $300^{\circ}$. A possible solution is to put the servo motor blind zone in the stern direction and add a constraint to (1):

$$
-\frac{5}{6} \pi<\psi_{s}<\frac{5}{6} \pi
$$

It is however easy to prove that for any value of $\psi_{s}$ there is a solution for both equations in (1).

\section{Extended Kalman Filter (EKF)}

After the analysis of different filter performances in navigation developed by the MDM Lab [10] [11] [12], the authors decided to use an EKF for the proposed application. The measurements coming from the sensors are thus processed by an EKF to obtain the vehicle state (position and orientation). According to the SNAME [13] notation, the pose (position and orientation) of an underwater robot is expressed with respect to the fixed-frame whereas its velocity (angular and rotational) is expressed with respect to the body-frame [14], by exploiting the following quantities:

$$
\begin{array}{ll}
\bar{\eta}=\left[\begin{array}{l}
\bar{\eta}_{1} \\
\bar{\eta}_{2}
\end{array}\right] \quad \bar{\eta}_{1}=\left[\begin{array}{l}
x \\
y \\
z
\end{array}\right] & \bar{\eta}_{2}=\left[\begin{array}{l}
\phi \\
\theta \\
\psi
\end{array}\right] \\
\bar{v}=\left[\begin{array}{c}
\bar{v}_{1} \\
\bar{v}_{2}
\end{array}\right] \quad \bar{v}_{1}=\left[\begin{array}{c}
u \\
v \\
w
\end{array}\right] & \bar{v}_{2}=\left[\begin{array}{l}
p \\
q \\
r
\end{array}\right] \\
\bar{\tau}=\left[\begin{array}{c}
\bar{\tau}_{1} \\
\bar{\tau}_{2}
\end{array}\right] \quad \bar{\tau}_{1}=\left[\begin{array}{c}
X \\
Y \\
Z
\end{array}\right] \quad \bar{\tau}_{2}=\left[\begin{array}{c}
K \\
M \\
N
\end{array}\right]
\end{array}
$$

where $\bar{\eta}$ is composed of position $\bar{\eta}_{1}$ [m] and orientation as RPY (roll, pitch and yaw) angles, collected in the vector $\bar{\eta}_{2}$ [rad] referred to the Earth-fixed frame. $\bar{v}$ contains the linear $\bar{v}_{1}[\mathrm{~m} / \mathrm{s}]$ and angular $\bar{v}_{2}[\mathrm{rad} / \mathrm{s}]$ velocity vectors referred to the body frame and $\bar{\tau}$ is composed of the forces $\bar{\tau}_{1}[\mathrm{~N}]$ and torques $\bar{\tau}_{2}[\mathrm{Nm}]$ applied to the vehicle referred to the body frame.

The discrete time dynamic system used to model the vehicle behaviour can be written as follows:

$$
\bar{x}_{k+1}=f\left(\bar{x}_{k}, \bar{u}_{k}\right)
$$


where the state vector $\bar{x}_{k}$ and the input signals vector $\bar{u}_{k}$ at the instant $k$ have been used; they are defined as follow:

$$
\begin{gathered}
\bar{x}_{k}=\left[\begin{array}{cccc}
\bar{\eta}_{1_{k}} & \bar{\eta}_{2_{k}} & \bar{v}_{1_{k}} & \dot{\bar{v}}_{1 k}
\end{array}\right]^{T} \\
\bar{u}_{k}=\left[\begin{array}{lll}
X_{k} & Y_{k} & Z_{k}
\end{array}\right]^{T}
\end{gathered}
$$

$f(\cdot)$ is a nonlinear function that can be written as in the following equations:

$$
\left\{\begin{array}{l}
\bar{\eta}_{1_{k+1}}=\bar{\eta}_{1_{k}}+\Delta_{k} J_{1}\left(\bar{\eta}_{2_{k}}\right) \bar{v}_{1_{k}} \\
\bar{\eta}_{2_{k+1}}=\bar{\eta}_{2_{k}} \\
\bar{v}_{1_{k+1}}=\bar{v}_{1_{k}}+\Delta_{k} \dot{\bar{v}}_{1_{k}} \\
\dot{\bar{v}}_{1_{k+1}}=g\left(\bar{x}_{k}, \bar{u}_{k}\right)
\end{array}\right.
$$

The $g(\cdot)$ nonlinear function can be obtained starting from the standard dynamic model of the vehicle, its equation of motion [14], with the following assumptions:

- the vehicle speed is always bounded at values low enough to consider negligible the contribution of the $C$ matrix;

- $\quad$ assuming that the vehicle dynamics develops mainly along his $X_{b}$ axis, the dynamics equations can be restricted to the $X_{b}$ axis.

This way, the motion equations can be considerably simplified, reaching the form in (7), where the components of $\dot{\bar{v}}_{1_{k+1}}$ vector are reported.

$$
\left\{\begin{aligned}
\dot{u}_{k+1} & =\frac{1}{m}\left(X-\frac{1}{2} \rho C_{r x} A_{f} u_{k}\left|u_{k}\right|\right) \\
\dot{v}_{k+1} & =0 \\
\dot{w}_{k+1} & =0
\end{aligned}\right.
$$

where $C_{r x}$ is the $X_{b}$ axis drag coefficient, $\rho$ is the fluid density and $A_{f}$ is the frontal area of the vehicle.

It is possible to write down the complete dynamical system adding the measurement equation:

$$
\left\{\begin{array}{l}
\bar{x}_{k+1}=f\left(\bar{x}_{k}, \bar{u}_{k}\right) \\
\bar{y}_{k}=h\left(\bar{x}_{k}, \bar{u}_{k}\right)
\end{array}\right.
$$

The measurement vector $\bar{y}_{k}$ contains the data collected by sensors:

$$
\bar{y}=\left[\begin{array}{c}
\bar{\eta}_{1 p} \\
\bar{\eta}_{2}
\end{array}\right]
$$

where $\bar{\eta}_{1 p}$ is the position referred to the pool frame.

$h\left(\bar{x}_{k}, \bar{u}_{k}\right)$ function is linear as in the following expression:

$$
\bar{y}_{k}=\left[\begin{array}{ll}
H 1 & O_{6 x 6}
\end{array}\right] \bar{x}_{k}
$$

where $H_{1}$ matrix is a block matrix defined as:

$$
H_{1}=\left[\begin{array}{cc}
H_{11} & 0_{3 x 3} \\
0_{3 x 3} & I_{3}
\end{array}\right]
$$

where $H_{11}$ [15] is:

$$
\left[\begin{array}{ccc}
\sin \psi_{p} & \cos _{p} & 0 \\
-\cos \psi_{p} & \sin \psi_{p} & 0 \\
0 & 0 & 1
\end{array}\right]
$$

If the covariance matrices of initial state, process noise and measurement noise are correctly defined, the filter ensures an accurate prediction of the vehicle state when the measurements are not available, performing correction when those measurements are instead collected.

\section{EXPERIMENTAL VALIDATIONS}

This part reports the validation procedures and the results obtained with FeelHippo vehicle; all the tests have been performed in the MDM Lab pool (described before). It is worth noting that the limited dimensions of the pool and its curved walls represent a very harsh acoustic environment, making sonar data very noisy.

This environment cannot thus reproduce properly sea conditions but it can be considered challenging for a first validation, as it is a worst case test environment.

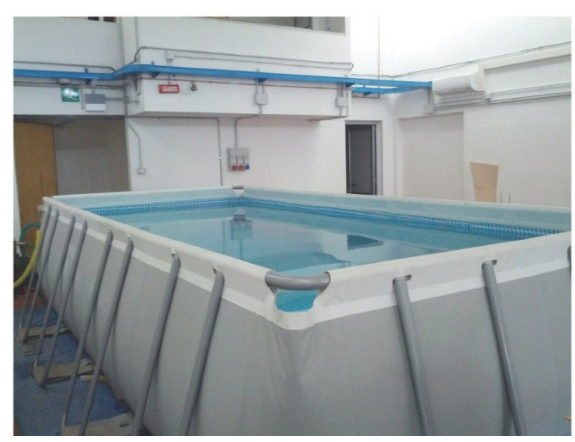

Fig. 6. Testing pool inside the MDM Lab (Pistoia, Italy)

A graphical interface has been developed in order to simplify the experimental tests. A screenshot is shown in Figure 7, and three different modalities can be set:

- Manual modality, where the operator can move each motor independently with a joystick;

- Autodepth modality, where yaw and depth PID ( Proportional, Integral and Derivative) controllers are enabled, but the references and the frontal thrust are given by the user using a joystick;

- $A U V$ modality, where the vehicle is completely autonomous and the user can only monitor its status.

The controllers parameters can also be tuned online at any time using this tool.

\section{A. Filter validation}

Once succeeded with parameters estimation it is possible to test the localization algorithm described in section II-D. In a first test a fixed forward thrust has been applied to the vehicle, controlling depth and yaw to be constant and the trajectory slightly oblique with respect to the pool (Figure 8). This way it is possible to test the filter performance w.r.t. all the three axes.

Figure 9 shows, in green, the behavior of estimated $y_{n}$ position compared with the sonar measurements, reported as red circles. The plot shows areas where the measurements 


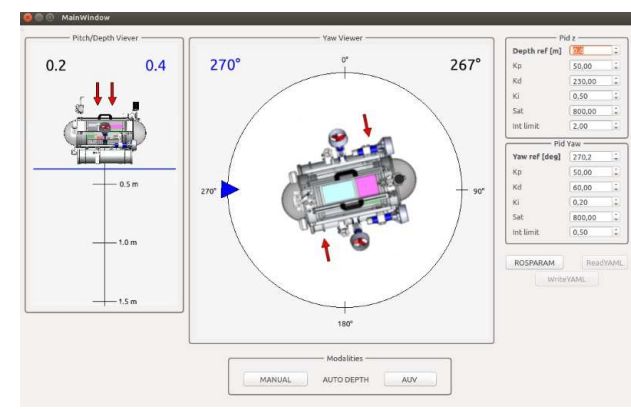

Fig. 7. Remote control software screenshot (taken from FeelHippo graphical interface)

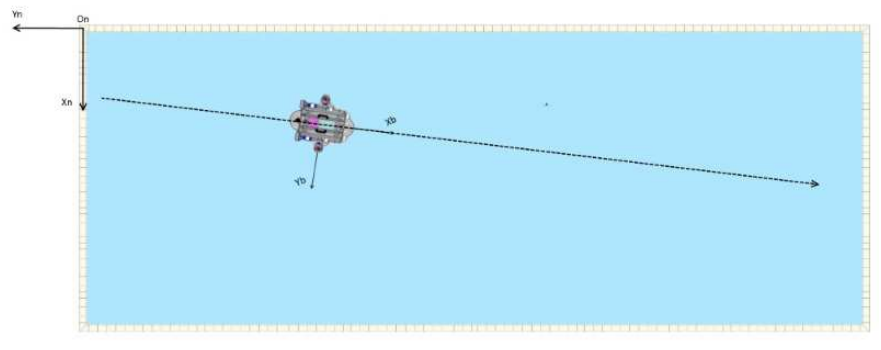

Fig. 8. Trajectory of the first navigation test inside the swimming pool

are not available and so only the prediction is working, and areas where a correction is performed using the measurements coming from the sonar. As concerns this axis the performances are very satisfying.

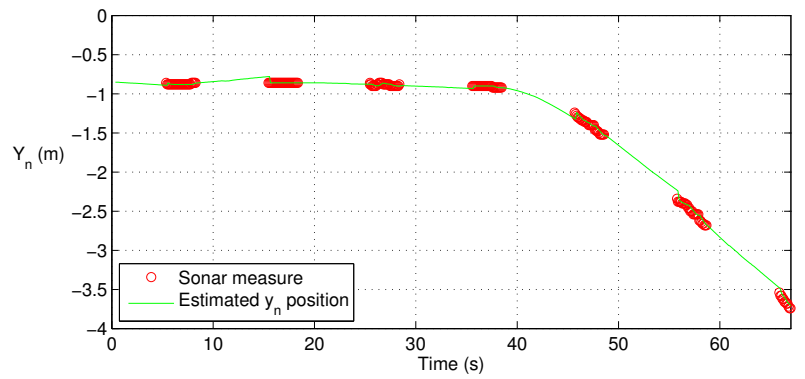

Fig. 9. Navigation filter behavior on y pool axis

Figure 10 shows, plotted as the previous one, the behavior of estimated $x_{n}$ position compared to the sonar data.

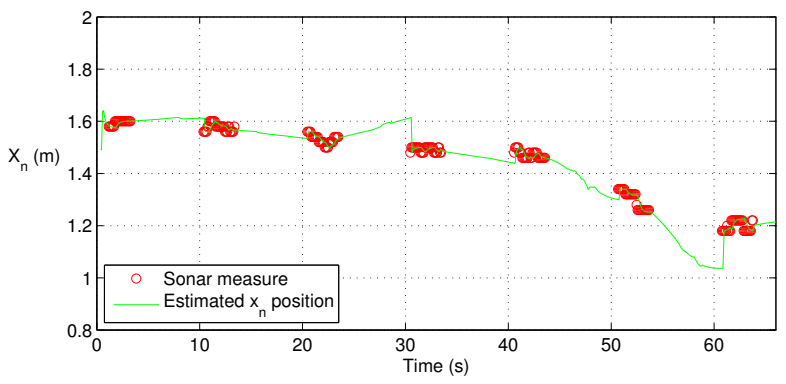

Fig. 10. Navigation filter behavior on $\mathrm{x}$ pool axis
In Figure 10 the strong measurement noise can be noticed, enhanced by the lower $L_{x}$ dimension of the pool, and by the slightly curved pool walls. This effect can be highlighted placing the sonar at a distance shorter than $1 \mathrm{~m}$ from the wall and observing the sonar raw data: this signal, reported in Figure 11, is featured by a high number of reflected waves, which make the readings very unreliable.

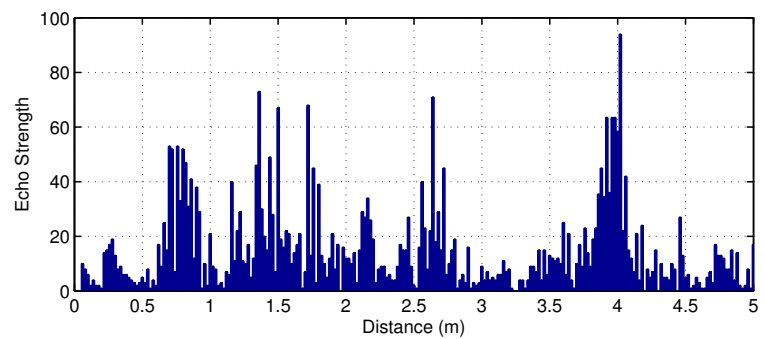

Fig. 11. Experimental test with sonar placed at $1 \mathrm{~m}$ distance from a wall.

\section{B. Autonomous navigation test}

The last phase of this work concerns an autonomous navigation test. The first step is to define a base task called "goToWaypoint", which consists on navigating from a starting point to a final one. This is composed by the following submissions executed sequentially:

- "changeOrientation", the first submission has the goal to let the vehicle assumes an orientation which brings the end point in front of it;

- "goToDepth", executed at the end of the first one, this submission brings the vehicle to a preset depth;

- "goToWP", where the vehicle, maintaining the bow pointed on the end point, is moved along the surge direction;

- "hovering", where the vehicle, reached the end point, maintain its position facing external disturbances until a new mission is assigned.

The test chosen for this work consists in a simple sequence of waypoints, which have to be reached sequentially using the above described procedure. The resulting desired trajectory is just a polyline which connects all the waypoints [10], [16].

Because of the small size of the pool, wide trajectories are not feasible, and thus a very simple set of waypoints has been chosen. The test consists on starting from the $A$ point (coordinates are $1.5 \mathrm{~m},-1.5 \mathrm{~m}, 0.7 \mathrm{~m}$ ), reaching a second point (coordinates $1.5 \mathrm{~m},-3 \mathrm{~m}, 0.7 \mathrm{~m}$ ) and going back to the first one. A $0.3 \mathrm{~m}$ tolerance has been chosen to detect the reached waypoint (Figure 12).

Figure 13 shows the estimated vehicle trajectory on the first leg $(A B)$ compared to the desired one.

In Figures 14 and 15 the position coordinates are reported separately against time during the whole test. As it can be seen, the vehicle starts from an estimated position of $x=1.2$ m $y=-1.55 \mathrm{~m}$, turning on the second point $x=1.54 \mathrm{~m}$, $y=-3.04 \mathrm{~m}$, to stop in $x=1.38 \mathrm{~m}, y=1.61 \mathrm{~m}$, complying the positions tolerance. 


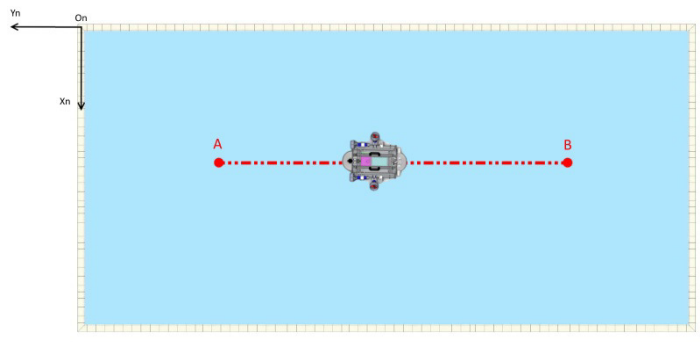

Fig. 12. Autonomous navigation test

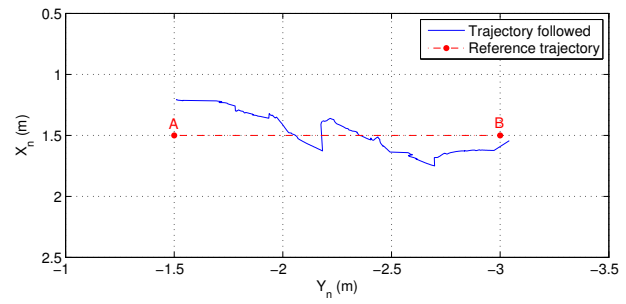

Fig. 13. Trajectory followed by the vehicle in the AB path

\section{CONCLUSION}

Usually, commercial underwater vehicles are very expensive. The challenge faced in this work was to find a good trade-off between performances, capabilities and costs considering an underwater localization algorithm exploiting a single beam sonar mounted on a low-cost underwater vehicle. FeelHippo AUV is a small cheap vehicle capable to house different payloads, such as cameras, acoustic modems, hydrophones, etc.. In this paper it has been demonstrated that, with a fine tuned Extended Kalman Filter, and the knowledge about the environment, it is possible to achieve a reliable estimation of the vehicle position in order to reach, in autonomous navigation, a sequence of prefixed waypoints. The results obtained show a great potential on

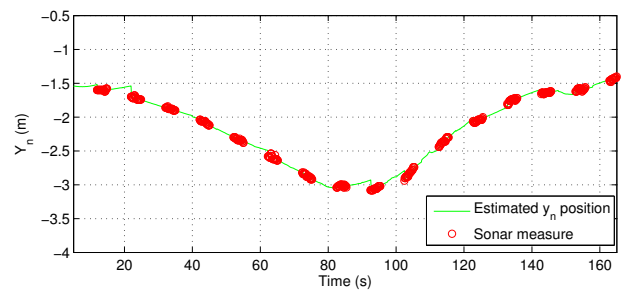

Fig. 14. $y_{n}$ coordinate vs time

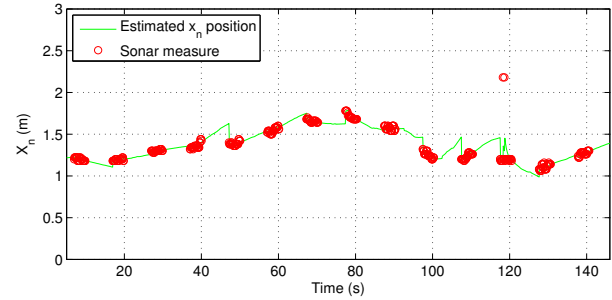

Fig. 15. $x_{n}$ coordinate vs time these techniques which should be further developed in the near future.

It is worth highlighting that the presented results have been achieved in a challenging environment: the swimming pool is not a simplified scenario, because of the water surface always close to the vehicle and because of its curved plastic walls. The authors are confident that the proposed results are satisfying and real scenarios will be investigated soon to confirm this statement.

\section{REFERENCES}

[1] F. Rourke, F. Boyle, and A. Reynolds, "Marine current energy devices: current status and possible future applications in ireland," Renewable and Sustainable Energy Reviews, 2010. vol. 14, Issue 3, p. 1026-1036.

[2] P. Rizzo, "NDE/SHM of underwater structures: a review," Advances in Science and Technology, 2013. vol. 83, p. 208-216.

[3] M. Jacobi, "Autonomous inspection of underwater structures," Robotics and Autonomous Systems, 2015. vol. 67, p. 80-86.

[4] B. Allotta, L. Pugi, F. Bartolini, A. Ridolfi, R. Costanzi, N. Monni, and J. Gelli, "Preliminary design and fast prototyping of an Autonomous Underwater Vehicle propulsion system," Proceedings of The Institution of Mechanical Engineers. Part M, Journal of Engineering for the Maritime Environment, 2015. vol. 229, p. 248-272.

[5] J. Carlton, Marine propellers and propulsion. 2nd edition, Elsevier, 2007.

[6] J. Lotz, L. M. Zurk, J. McNames, and J. L. Ecochard, "Coral fish shoal detection from acoustic echograms," in Proceedings of the OCEANS'07, Vancouver, BC, 2007.

[7] T. Ellis, J. McNames, L. Zurk, J. Lotz, and J. L. Ecochard, "Echosounder depth tracking with the Extended Kalman Filter," in Proceedings of the OCEANS'07, Vancouver, BC, 2007.

[8] N. Yazdi, F. Ayazi, and K. Najafi, "Micromachined inertial sensors," Proceedings of the IEEE, vol. 86, Issue 8, 2002.

[9] B. Allotta, R. Costanzi, F. Fanelli, N. Monni, and A. Ridolfi, "Single axis FOG aided attitude estimation algorithm for mobile robots," Mechatronics, 2015. vol. 30, p. 158-173.

[10] B. Allotta, R. Costanzi, N. Monni, L. Pugi, and A. Ridolfi, "Design and simulation of an Autonomous Underwater Vehicle," in Proceedings of the European Congress on Computational Methods in Applied Sciences and Engineering (ECCOMAS), Vienna, Austria, Sept. 10-14, 2012.

[11] B. Allotta, L. Chisci, R. Costanzi, F. Fanelli, C. Fantacci, E. Meli, A. Ridolfi, A. Caiti, F. D. Corato, and D. Fenucci, "A comparison between EKF-based and UKF-based navigation algorithms for AUVs localization," in Proceedings of the OCEANS15 MTS/IEEE GENOVA, Genova, Italy, May 2015.

[12] B. Allotta, A. Caiti, R. Costanzi, F. Fanelli, D. Fenucci, E. Meli, and A. Ridolfi, "A new AUV navigation system exploiting Unscented Kalman Filter," Elsevier Ocean Engineering, 2016. vol. 113, p. 121132.

[13] SNAME, "Nomenclature for treating the motion of a submerged body through a fluid," 1950. Society of Naval Architects and Marine Engineers, Technical Report Bulletin 1-5, New York.

[14] T. I. Fossen, Guidance and control of ocean vehicles. John Wiley \& Sons, Ltd, 1994.

[15] B. Siciliano and O. Khatib, Handbook of Robotics. Springer Handbooks, Napoli and Stanford, 2008.

[16] M. Breivik and T. I. Fossen, "Guidance-based path following for autonomous underwater vehicles," in Proceedings of the OCEANS'05, Washington D.C., USA, 2005. 